\title{
BMJ Open Scoping review of evidence for managing postnatal hypoglycaemia
}

\author{
Jennifer Horwitz, ${ }^{1}$ Linda Mardiros (1) , ${ }^{1}$ Ahmed Musa (1) , ${ }^{1}$ Vivian A Welch (D) ,,3 \\ Amanda Hodgson (D) , ${ }^{4}$ Michael Narvey, ${ }^{5}$ Andrea Ghazzawi, ${ }^{6}$ Beverley Shea, ${ }^{2,3}$ \\ Michael Saginur ${ }^{7,8}$
}

To cite: Horwitz J, Mardiros L, Musa A, et al. Scoping review of evidence for managing postnata hypoglycaemia. BMJ Open 2022;12:e053047. doi:10.1136/ bmjopen-2021-053047

- Prepublication history and additional supplemental material for this paper are available online. To view these files, please visit the journal online (http://dx.doi.org/10.1136/ bmjopen-2021-053047).

Received 14 May 2021 Accepted 30 December 2021

Check for updates

(c) Author(s) (or their employer(s)) 2022. Re-use permitted under CC BY-NC. No commercial re-use. See rights and permissions. Published by BMJ.

${ }^{1}$ Faculty of Medicine, University of Ottawa, Ottawa, Ontario,

Canada

${ }^{2}$ Bruyère Research Institute, Ottawa, Ontario, Canada

${ }^{3}$ School of Epidemiology and Public Health, Faculty of Medicine, University of Ottawa, Ottawa, Ontario, Canada ${ }^{4}$ University of Ottawa Library, Ottawa, Ontario, Canada

${ }^{5}$ Department of Pediatrics, University of Manitoba, Winnipeg, Manitoba, Canada

${ }^{6}$ Telfer School of Management, University of Ottawa, Ottawa, Ontario, Canada

${ }^{7}$ Montfort Hospital, Ottawa,

Ontario, Canada

${ }^{8}$ Department of Pediatrics, Faculty of Medicine, University of Ottawa, Ottawa, Ontario, Canada

Correspondence to Dr Michael Saginur; michaelsaginur@montfort.on.ca

\section{ABSTRACT}

Objectives To identify what is known empirically about the screening, treatment and harm of exposure to neonatal hypoglycaemia.

Design Scoping review that applied a preregistered protocol based on established frameworks.

Data sources Medline and Embase, up to 12 May 2020.

Study selection Comparative and case-series studies, as well as guidelines, published in English or French, on the topic of immediate inpatient postnatal glucose screening in newborns.

Data gathering Article selection and characterisation were performed in duplicate using predefined data extraction forms specific to primary studies and guidelines. Results 12 guidelines and 74 primary studies were included. A neurodevelopmental outcome was primary in 32 studies: 30 observational studies followed up posthypoglycaemic, and the 2 intervention studies included 1 randomised controlled trial (RCT) about treatment thresholds. Three other RCTs assessed dextrose gel (two) and oral sucrose (one). 12 of 30 studies that evaluated non-neurodevelopmental primary outcomes were intervention studies. Only one cohort study compared outcomes in screened vs unscreened newborns. The guidelines did not arrive at a consensus definition of postnatal hypoglycaemic, and addressed potential harms of screening more often than primary studies.

Conclusions The primary literature that informs hypoglycaemia screening is a series of studies that relate neurodevelopmental outcomes to postnatal hypoglycaemia. Further research is needed to better define an optimal threshold for hypoglycaemia that warrants intervention, based on long-term neurodevelopmental outcomes and a better delineation of potential screening harms.

\section{INTRODUCTION}

Hypoglycaemia screening in at-risk babies is a well-accepted practice in the third of babies with risk factors ${ }^{1-5}$ however, screening approaches vary in the context of ongoing controversy around diagnosis and treatment. ${ }^{6}$ Screening for postnatal hypoglycaemia aims to avoid acute and long-term hypoglycaemia complications such as seizures and neurodevelopmental delay; ${ }^{7}$ however, recognition that screening is not entirely benign ${ }^{8}$ means asymptomatic, low-risk babies are not

\section{Strengths and limitations of this study}

Broad literature search.

- Transparent and systematic application of established methodological frameworks.

- As a scoping study, did not consider study results or assess the quality of the evidence, so does not provide synthesised answers to inform clinical decision making.

- The analysis considered only primary study outcomes as opposed to all study outcomes.

- Language restrictions may have missed a small number of studies.

screened. Ongoing debate around the justification for newborn screening in general cites concerns around costs, the nature of subsequent treatment and consent. ${ }^{9}$ There is no consensus regarding the threshold for hypoglycaemia risk that warrants screening, nor the hypoglycaemia threshold that requires intervention. Ideally, both values would be informed by a balance of demonstrated benefits and harms.

A recent systematic review on neonatal hypoglycaemia (NH) and neurodevelopment found that $\mathrm{NH}$ was associated with impairment that becomes fully apparent after school age is reached. ${ }^{10}$ However, the latest broad $\mathrm{NH}$-evidence review is more than a decade old. ${ }^{11}$

This scoping review provides an updated description of the literature on $\mathrm{NH}$ harms, and NH screening and treatment. The objectives of this scoping review are to better understand the basis in evidence for different approaches to managing $\mathrm{NH}$, and guide future $\mathrm{NH}$ research. The scoping-review approach allowed for a characterisation of the relevant supporting evidence, as well as gaps in the literature. Guideline review provided an overview of different approaches to managing $\mathrm{NH}$, which further contextualised this evidence summary. 


\section{METHODS}

The scoping literature review used established methodological frameworks. ${ }^{12} 1314$

\section{Patient and public involvement}

Patients and the public were not involved in this study's design, conduct or dissemination plans.

\section{Search strategy}

The peer-reviewed search strategy ${ }^{15}$ searched MEDLINE and Embase via the Ovid platform on 12 May 2020. The main search concept was $\mathrm{NH}$, along with search filters for systematic reviews, guidelines, clinical trials and observational studies, built on established filters from the Canadian Agency for Drugs and Technologies in Health. ${ }^{16}$ Based on findings from preliminary searches for systematic reviews and guidelines, the final search limited systematic reviews from 2005 to present, but applied no other date limits. The search was limited to English or French language publications, and omitted conference abstracts. Reference lists of systematic reviews and guidelines were reviewed manually to identify potentially relevant articles.

\section{Study selection}

Three reviewers (AM, LM and $\mathrm{JH}$ ) independently screened study titles and abstracts in duplicate. Disagreements around eligibility could be resolved by the third reviewer: and if needed, by a fourth (MS).

Included studies involved human newborn glucose screening in the perinatal hospital setting, with onset of hypoglycaemia at less than 48 hours of life. Potential interventions and exposures included hypoglycaemia, screening for hypoglycaemia, and treatment for hypoglycaemia. Eligible outcomes were related to either health (eg, seizures) or resource use (eg, length of stay).

All comparative study designs were included: that is, RCTs, cohorts, case-controls, pre-post and interrupted time-series studies. Since we anticipated a limited amount of research on $\mathrm{NH}$ with non-neurodevelopmental outcomes, case studies with greater than five subjects and non-neurodevelopmental outcomes were included. Thus, case series (where no exposure-based comparison was possible) with both a neurodevelopmental and a non-neurodevelopmental outcome were included; however, only primary study outcomes were considered. All other case series' were excluded. Primary outcomes were as specified in the study reports: based on the data extractors' impressions when unspecified.

We envisioned four main categories of primary comparative studies:

1. Developmental outcomes of hypoglycaemia.

2. Non-developmental hypoglycaemia-related outcomes.

3. Outcomes of hypoglycaemic treatment versus no treatment.

4. Outcomes of hypoglycaemic screening (routine screening for $\mathrm{NH}$ based on predefined risk categories).

We also included national and international guidelines, programmes of care, treatment protocols, policy

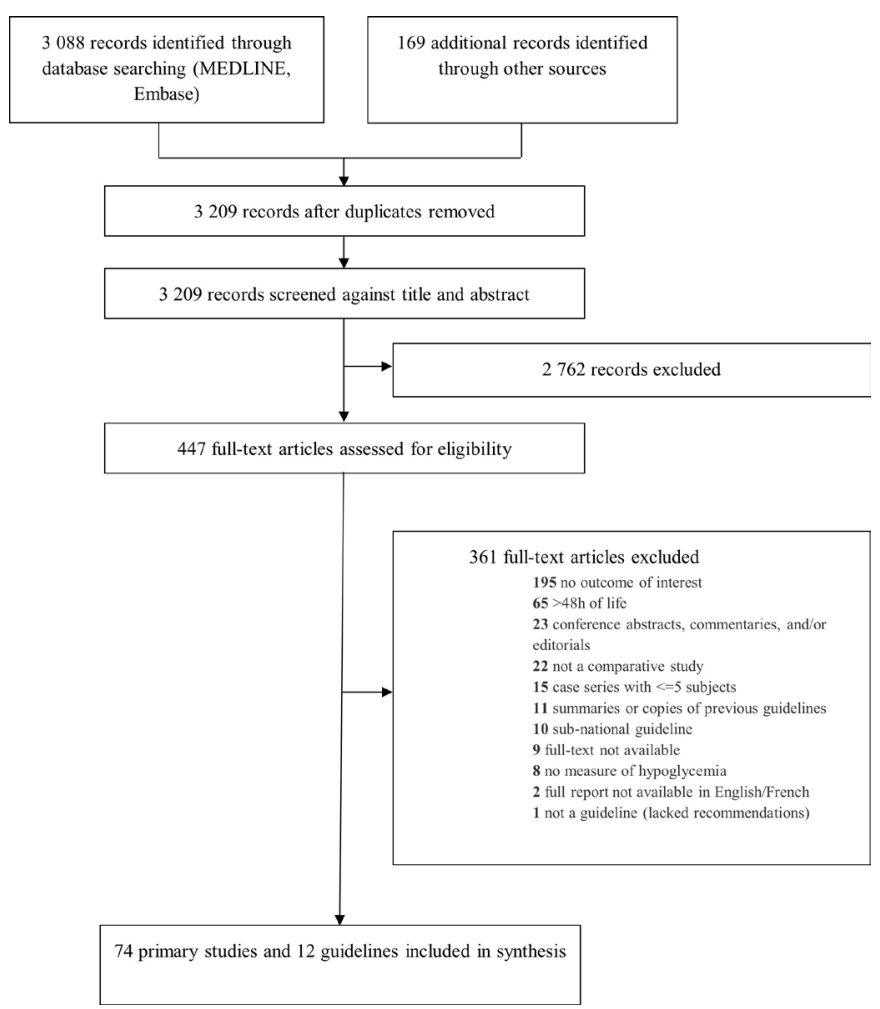

Figure 1 Screening and selection process.

statements and position statements that addressed $\mathrm{NH}$. We excluded those lacking treatment recommendations and excluded summaries or copies of previous guidelines. ${ }^{17}$ We excluded conference abstracts, commentaries, editorials and articles not available in English or French.

\section{Data management software}

Endnote tracked references and excluded duplicate articles. Covidence tracked article inclusions and exclusions. ${ }^{18}$ We entered extracted data into Google sheets, using preset categories as much as possible.

\section{Data extraction}

Predefined data extraction forms specific to primary studies and guidelines were edited and used after pilot extraction. As is the norm for scoping reviews, we did not evaluate the risk of bias in the study findings, though we did employ the AGREE-II question about guidelines' use of systematic methods to search for evidence. ${ }^{19}$ The three data collectors met regularly and reviewed their work on a 5\% sample at each new step. Content analysis was used to analyse the data and identify emergent themes.

\section{RESULTS}

\section{Primary studies}

We included 74 primary studies (figure 1 and online supplemental table 1), primarily from the Organisation for Economic Co-operation and Development countries ('OECD'; $80 \%$ of studies). Their median publication date was 2012 (mean 2005; range 1965-2020). Threequarters were controlled observational studies (table 1). 
Table 1 Characteristics of primary studies

\begin{tabular}{|c|c|}
\hline Total primary studies & $\mathrm{n}=74$ \\
\hline \multicolumn{2}{|l|}{ Sample size: median, mean } \\
\hline Study group $(n=71)$ & $58.5,529.0$ \\
\hline Control group $(n=42)$ & $70.0,2579.9$ \\
\hline \multicolumn{2}{|l|}{ Design $(n=74)$} \\
\hline Cohort, prospective & $47 \%$ \\
\hline Cohort, retrospective & $30 \%$ \\
\hline Case series & $9 \%$ \\
\hline $\mathrm{RCT}$ & $5 \%$ \\
\hline Pre-post & $5 \%$ \\
\hline Case-control & $3 \%$ \\
\hline \multicolumn{2}{|l|}{ Funding source $(n=31)$} \\
\hline Non-profit & $68 \%$ \\
\hline Industry & $32 \%$ \\
\hline Premature $(n=43)$ & $37 \%$ \\
\hline Gestational age, mean weeks $(n=45)$ & 36.9 \\
\hline Birth weight: mean $(n=45)$ & $2773.8 \mathrm{~g}$ \\
\hline \multicolumn{2}{|l|}{ Basis of hypoglycaemia diagnosis $(n=71)$} \\
\hline Glucose value alone & $86 \%$ \\
\hline Glucose value and clinical signs & $14 \%$ \\
\hline \multicolumn{2}{|l|}{ Testing method $(n=42)$} \\
\hline Laboratory & $50 \%$ \\
\hline Bedside & $33 \%$ \\
\hline Continuous glucose monitoring & $7 \%$ \\
\hline Laboratory and bedside & $7 \%$ \\
\hline $\begin{array}{l}\text { Laboratory and continuous glucose } \\
\text { monitoring }\end{array}$ & $2 \%$ \\
\hline \multicolumn{2}{|l|}{ Sample $(n=13)$} \\
\hline Capillary & $85 \%$ \\
\hline Venous & $15 \%$ \\
\hline Both & $0 \%$ \\
\hline \multicolumn{2}{|l|}{ Sample assayed $(n=11)$} \\
\hline Plasma & $55 \%$ \\
\hline Whole blood & $45 \%$ \\
\hline \multicolumn{2}{|l|}{ Screening Indication $(n=75)^{*}$} \\
\hline Infant of diabetic mother & $37 \%$ \\
\hline Small for gestational age & $33 \%$ \\
\hline Large for gestational age & $28 \%$ \\
\hline Premature birth & $33 \%$ \\
\hline \multicolumn{2}{|l|}{ Other $(n=52)$} \\
\hline No reason for screening provided & $69 \%$ \\
\hline Symptomatic hypoglycaemia & $21 \%$ \\
\hline 'High-risk infants' & $13 \%$ \\
\hline Blanket screening policy (all newborns) & $12 \%$ \\
\hline Asphyxia & $10 \%$ \\
\hline Sepsis & $10 \%$ \\
\hline Seizures & $10 \%$ \\
\hline Hypoxic ischaemic & $10 \%$ \\
\hline
\end{tabular}

Continued

\begin{tabular}{cc}
\hline Table 1 Continued & \\
\hline Encephalopathy & $8 \%$ \\
Poor feeding & $8 \%$ \\
Respiratory distress & $8 \%$ \\
Low Apgar score & $6 \%$
\end{tabular}

*Some studies counted in multiple categories. $\mathrm{RCT}$, randomised controlled trial.

Babies were typically early term (online supplemental figure 1) and studies' subjects on average were $56 \%$ male $(\mathrm{n}=60)$. In $86 \%$, hypoglycaemia diagnoses were based on glucose levels alone. The most common hypoglycaemia threshold was $2.6 \mathrm{mmol} / \mathrm{L}$ (mean $2.22 \mathrm{mmol} / \mathrm{L}$, median $2.4 \mathrm{mmol} / \mathrm{L}, \mathrm{n}=67)$. Blood glucose was measured in the lab in $50 \%$ of reporting studies, at the bedside in $33.3 \%$, both in the lab and at bedside in $7 \%$ and from continuous glucose monitors in 7\%. Few studies reported whether sampling was capillary or venous; whole blood or plasma.

\section{Neurodevelopmental studies}

The 32 studies with a primary neurodevelopmental outcome were published between 1965 and 2020 (median 2010, mean 2002). Of these, 2 were interventional (1 randomised) and 30 observational (table 2). The mean sample size was 320; the total was 10253 . Study hypoglycaemia thresholds ranged from 1.11 to $2.8 \mathrm{mmol} / \mathrm{L}$ (mean 2.19, median 2.3, mode 2.6). Hypoglycaemia diagnosis was also based on symptoms in 4 of 32 reporting studies (with a mean publication date of 1984 , and a mean hypoglycaemic threshold of $1.7 \mathrm{mmol} / \mathrm{L}$ ).

Although three of the studies screened all newborns for hypoglycaemia, 19 of the studies screened newborns specifically for at least one of the most common indications: prematurity, small for gestational age (GA), large for GA or maternal diabetes. The other studies focused on babies who were compromised or potentially symptomatic.

The Bayley Scales of Infant Development III, Griffiths Scale of Mental Development, and Wechsler Intelligence Scale for Children were used most frequently (online supplemental table 2). Studies also addressed specific domains such as vision. Follow-up duration averaged 5.5 years, but spanned a range of 20 years; $12 \%$ of studies addressed the first year of life, $59 \%$ early childhood (2-5 years), 21\% mid-childhood (6-11 years) and 3\% early adulthood (18 years or older).

The two studies of treatment included a randomised controlled trial (RCT) of dextrose-gel treatment thresholds and psychomotor development at 18 months, and a prospective cohort study that related maternal glucose therapy in labour to neurobehaviour at 1-2 hours of age. Twenty-seven comparative exposure studies and three case series investigated neurodevelopmental outcomes with postnatal hypoglycaemia. One investigated glucose variation in the first 24 hours in hypoxic-ischaemic encephalopathy and neurodevelopment. 
Open access

Table 2 Characteristics of primarily neurodevelopmental and non-neurodevelopmental studies

\section{Primary studies: neurodevelopmental}

\begin{tabular}{|c|c|c|c|}
\hline \multicolumn{2}{|l|}{ Primary studies: neurodevelopmental } & \multicolumn{2}{|l|}{ Primary studies: non-neurodevelopmental } \\
\hline Total & $\mathrm{N}=32$ & Total & $\mathrm{N}=\mathbf{3 0}$ \\
\hline Design & & Design & \\
\hline Cohort, prospective & $56 \%$ & Cohort, retrospective & $40 \%$ \\
\hline Cohort, retrospective & $25 \%$ & Cohort, prospective & $33 \%$ \\
\hline Case series & $9 \%$ & Pre-post & $13 \%$ \\
\hline Case-control & $6 \%$ & RCT & $10 \%$ \\
\hline $\mathrm{RCT}$ & $3 \%$ & Case series & $3 \%$ \\
\hline Indications for screening & $(n=32)^{\star}$ & Exposures and study design & $(n=17)$ \\
\hline Premature & $40 \%$ & Hypoglycaemic neonates & $47 \%$ \\
\hline Small for gestational age & $34 \%$ & Other exposures & $53 \%$ \\
\hline Large for gestational age & $34 \%$ & Neonatal seizures & $18 \%$ \\
\hline Infant of diabetic mother & $28 \%$ & Infants of diabetic mothers & $6 \%$ each \\
\hline Others: & & Hypoglycaemia risk criteria & \\
\hline Hypoxic ischaemic injury & $6 \%$ & Hypoglycaemia and acidemia & \\
\hline Symptomatic hypoglycaemia & $6 \%$ & Blood glucose fluctuation & \\
\hline At risk for neonatal encephalopathy & $3 \%$ & Hypoxic-ischaemic encephalopathy & \\
\hline Neonatal asphyxia† & $3 \%$ & Screening for asymptomatic hypoglycaemia & \\
\hline Neonatal convulsions & $3 \%$ & & \\
\hline $\begin{array}{l}\text { Other conditions (eg, poor feeding, respiratory } \\
\text { distress, sepsis, intrauterine growth retardation, } \\
\text { pregnancy-induced hypertension) }\end{array}$ & $16 \%$ & & \\
\hline Screen all newborns & $9 \%$ & & \\
\hline Outcomes & $(n=32)^{\star}$ & Outcomes & $(\mathrm{n}=30)^{*}$ \\
\hline Neurodevelopmental impairment & $75 \%$ & Admission rate & $27 \%$ \\
\hline Literacy and test scores & & Seizures & $17 \%$ \\
\hline Establishment of neurodevelopmental & $9 \%$ & Brain injury & $13 \%$ \\
\hline diagnoses $\ddagger$ & $9 \%$ & Early frequent breastfeeding to prevent hypoglycaemia & $13 \%$ \\
\hline Executive dysfunction & $6 \%$ & Breastfeeding rate & $10 \%$ \\
\hline \multirow[t]{17}{*}{ Visual impairment } & $6 \%$ & Cost of treatment & $7 \%$ \\
\hline & $9 \%$ & Mortality & $3 \%$ each \\
\hline & $6 \%$ & Complications of continuous glucose monitoring & \\
\hline & $6 \%$ & Cardiothoracic abnormality & \\
\hline & & Inborn error of metabolism & \\
\hline & & Growth parameter abnormality & \\
\hline & & Intravenous dextrose treatment & \\
\hline & & Dextrose gel treatment & \\
\hline & & Intravenous bolus treatment & \\
\hline & & Hypoglycaemic relapse & \\
\hline & & Interventions and study designs & $(n=12)^{*}$ \\
\hline & & $40 \%$ dextrose gel§ & $50 \%$ \\
\hline & & Altered breastfeeding & $33 \%$ \\
\hline & & Intravenous Infusion & $25 \%$ \\
\hline & & Toolkit creation§ & $8 \%$ \\
\hline & & Skin to skin & $8 \%$ \\
\hline & & Oral sucrose & $8 \%$ \\
\hline
\end{tabular}

\begin{tabular}{|c|c|c|c|}
\hline \multicolumn{2}{|l|}{ Primary studies: neurodevelopmental } & \multicolumn{2}{|l|}{ Primary studies: non-neurodevelopmental } \\
\hline Total & $\mathrm{N}=32$ & Total & $\mathrm{N}=\mathbf{3 0}$ \\
\hline Design & & Design & \\
\hline Cohort, prospective & $56 \%$ & Cohort, retrospective & $40 \%$ \\
\hline Cohort, retrospective & $25 \%$ & Cohort, prospective & $33 \%$ \\
\hline Case series & $9 \%$ & Pre-post & $13 \%$ \\
\hline Case-control & $6 \%$ & RCT & $10 \%$ \\
\hline $\mathrm{RCT}$ & $3 \%$ & Case series & $3 \%$ \\
\hline Indications for screening & $(n=32)^{\star}$ & Exposures and study design & $(n=17)$ \\
\hline Premature & $40 \%$ & Hypoglycaemic neonates & $47 \%$ \\
\hline Small for gestational age & $34 \%$ & Other exposures & $53 \%$ \\
\hline Large for gestational age & $34 \%$ & Neonatal seizures & $18 \%$ \\
\hline Infant of diabetic mother & $28 \%$ & Infants of diabetic mothers & $6 \%$ each \\
\hline Others: & & Hypoglycaemia risk criteria & \\
\hline Hypoxic ischaemic injury & $6 \%$ & Hypoglycaemia and acidemia & \\
\hline Symptomatic hypoglycaemia & $6 \%$ & Blood glucose fluctuation & \\
\hline At risk for neonatal encephalopathy & $3 \%$ & Hypoxic-ischaemic encephalopathy & \\
\hline Neonatal asphyxia† & $3 \%$ & Screening for asymptomatic hypoglycaemia & \\
\hline Neonatal convulsions & $3 \%$ & & \\
\hline $\begin{array}{l}\text { Other conditions (eg, poor feeding, respiratory } \\
\text { distress, sepsis, intrauterine growth retardation, } \\
\text { pregnancy-induced hypertension) }\end{array}$ & $16 \%$ & & \\
\hline Screen all newborns & $9 \%$ & & \\
\hline Outcomes & $(n=32)^{\star}$ & Outcomes & $(\mathrm{n}=30)^{*}$ \\
\hline Neurodevelopmental impairment & $75 \%$ & Admission rate & $27 \%$ \\
\hline Literacy and test scores & & Seizures & $17 \%$ \\
\hline Establishment of neurodevelopmental & $9 \%$ & Brain injury & $13 \%$ \\
\hline diagnoses $\ddagger$ & $9 \%$ & Early frequent breastfeeding to prevent hypoglycaemia & $13 \%$ \\
\hline Executive dysfunction & $6 \%$ & Breastfeeding rate & $10 \%$ \\
\hline \multirow[t]{17}{*}{ Visual impairment } & $6 \%$ & Cost of treatment & $7 \%$ \\
\hline & $9 \%$ & Mortality & $3 \%$ each \\
\hline & $6 \%$ & Complications of continuous glucose monitoring & \\
\hline & $6 \%$ & Cardiothoracic abnormality & \\
\hline & & Inborn error of metabolism & \\
\hline & & Growth parameter abnormality & \\
\hline & & Intravenous dextrose treatment & \\
\hline & & Dextrose gel treatment & \\
\hline & & Intravenous bolus treatment & \\
\hline & & Hypoglycaemic relapse & \\
\hline & & Interventions and study designs & $(n=12)^{*}$ \\
\hline & & $40 \%$ dextrose gel§ & $50 \%$ \\
\hline & & Altered breastfeeding & $33 \%$ \\
\hline & & Intravenous Infusion & $25 \%$ \\
\hline & & Toolkit creation§ & $8 \%$ \\
\hline & & Skin to skin & $8 \%$ \\
\hline & & Oral sucrose & $8 \%$ \\
\hline
\end{tabular}

Outcomes $(n=32)^{*}$

\begin{tabular}{|c|c|c|c|}
\hline \multicolumn{2}{|l|}{ Primary studies: neurodevelopmental } & \multicolumn{2}{|l|}{ Primary studies: non-neurodevelopmental } \\
\hline Total & $\mathrm{N}=32$ & Total & $\mathrm{N}=\mathbf{3 0}$ \\
\hline Design & & Design & \\
\hline Cohort, prospective & $56 \%$ & Cohort, retrospective & $40 \%$ \\
\hline Cohort, retrospective & $25 \%$ & Cohort, prospective & $33 \%$ \\
\hline Case series & $9 \%$ & Pre-post & $13 \%$ \\
\hline Case-control & $6 \%$ & RCT & $10 \%$ \\
\hline $\mathrm{RCT}$ & $3 \%$ & Case series & $3 \%$ \\
\hline Indications for screening & $(n=32)^{\star}$ & Exposures and study design & $(n=17)$ \\
\hline Premature & $40 \%$ & Hypoglycaemic neonates & $47 \%$ \\
\hline Small for gestational age & $34 \%$ & Other exposures & $53 \%$ \\
\hline Large for gestational age & $34 \%$ & Neonatal seizures & $18 \%$ \\
\hline Infant of diabetic mother & $28 \%$ & Infants of diabetic mothers & $6 \%$ each \\
\hline Others: & & Hypoglycaemia risk criteria & \\
\hline Hypoxic ischaemic injury & $6 \%$ & Hypoglycaemia and acidemia & \\
\hline Symptomatic hypoglycaemia & $6 \%$ & Blood glucose fluctuation & \\
\hline At risk for neonatal encephalopathy & $3 \%$ & Hypoxic-ischaemic encephalopathy & \\
\hline Neonatal asphyxia† & $3 \%$ & Screening for asymptomatic hypoglycaemia & \\
\hline Neonatal convulsions & $3 \%$ & & \\
\hline $\begin{array}{l}\text { Other conditions (eg, poor feeding, respiratory } \\
\text { distress, sepsis, intrauterine growth retardation, } \\
\text { pregnancy-induced hypertension) }\end{array}$ & $16 \%$ & & \\
\hline Screen all newborns & $9 \%$ & & \\
\hline Outcomes & $(n=32)^{\star}$ & Outcomes & $(\mathrm{n}=30)^{*}$ \\
\hline Neurodevelopmental impairment & $75 \%$ & Admission rate & $27 \%$ \\
\hline Literacy and test scores & & Seizures & $17 \%$ \\
\hline Establishment of neurodevelopmental & $9 \%$ & Brain injury & $13 \%$ \\
\hline diagnoses $\ddagger$ & $9 \%$ & Early frequent breastfeeding to prevent hypoglycaemia & $13 \%$ \\
\hline Executive dysfunction & $6 \%$ & Breastfeeding rate & $10 \%$ \\
\hline \multirow[t]{17}{*}{ Visual impairment } & $6 \%$ & Cost of treatment & $7 \%$ \\
\hline & $9 \%$ & Mortality & $3 \%$ each \\
\hline & $6 \%$ & Complications of continuous glucose monitoring & \\
\hline & $6 \%$ & Cardiothoracic abnormality & \\
\hline & & Inborn error of metabolism & \\
\hline & & Growth parameter abnormality & \\
\hline & & Intravenous dextrose treatment & \\
\hline & & Dextrose gel treatment & \\
\hline & & Intravenous bolus treatment & \\
\hline & & Hypoglycaemic relapse & \\
\hline & & Interventions and study designs & $(n=12)^{*}$ \\
\hline & & $40 \%$ dextrose gel§ & $50 \%$ \\
\hline & & Altered breastfeeding & $33 \%$ \\
\hline & & Intravenous Infusion & $25 \%$ \\
\hline & & Toolkit creation§ & $8 \%$ \\
\hline & & Skin to skin & $8 \%$ \\
\hline & & Oral sucrose & $8 \%$ \\
\hline
\end{tabular}

*Some studies counted in multiple categories.

†Defined as two or more of: initial capillary or arterial $\mathrm{pH}<7.1$, Apgar score $<5$ at $5 \mathrm{~min}$, initial capillary or arterial lactate $>7 \mathrm{mmol} / \mathrm{L}$, abnormal neurology or clinical seizures.

‡For example, cerebral palsy, autism, mental retardation.

§One study had an unspecified intervention threshold.

$\mathrm{RCT}$, randomised controlled trial. 


\section{Non-neurodevelopmental studies}

Studies with a primary non-neurodevelopmental outcome were published between 1975 and 2020 (median 2016, mean 2011; 73\% OECD). Thirty studies evaluated the relationship between $\mathrm{NH}$ and non-neurodevelopmental primary outcomes; 11 evaluated the relationship as a secondary outcome. Their mean sample sizes were 973 and 276 for the treatment and control groups. Study hypoglycaemic thresholds ranged from 1.6 to $2.6 \mathrm{mmol} / \mathrm{L}$ (mean 2.34, median 2.4, mode 2.6); one study adjusted the threshold for birth weight. Twenty-one studies were cohort studies, and four were RCTs (table 2).

Aside from the case-series study, 17 studies explored exposures and 12 assessed different interventions (table 2). The following significant outcomes were measured only in observational studies: mortality, brain injury, inborn errors of metabolisms, and growth parameter abnormalities. Among the 17 exposure studies, outcomes included admission rates, brain injury, seizures, mortality and diagnosed inborn error of metabolism. One study of continuous glucose monitoring considered the parental perspective on such monitoring.

Six of the intervention studies were on carbohydrate gels: one oral sucrose; three intravenous infusions; four breastfeeding interventions; one toolkit creation and one skin to skin. The interventions that were assessed with RCTs were dextrose gels (two), oral sucrose (one) and altered intravenous infusion (one), with outcomes that included admission rates, treatment cost and intravenous treatment.

The follow-up duration for the non-neurodevelopmental studies was detailed in eight studies, and ranged from 7 days to 21 years. Interestingly, neonates with suspected brain injury outcomes were followed for the shortest duration: 7 days in two studies, 2 years in another and 5 years in the last study. In contrast, the follow-up duration for seizures outcomes was 1 year in one study, 2 years in one study and 21 years in another study.

\section{Both neurodevelopmental and non-neurodevelopmental outcomes}

Eight cohort studies and three case series studies focused equally on neurodevelopmental and nonneurodevelopmental outcomes. Their themes were outcomes of $\mathrm{NH}(\mathrm{n}=7)$; glucose profiles with therapeutic hypothermia for hypoxic-ischaemic encephalopathy $(\mathrm{n}=1)$; NH-related events such as seizures $(\mathrm{n}=2)$; a central nervous system/ocular outcomes $(n=1)$; and differentiation between symptomatic and asymptomatic hypoglycaemic $(n=2)$.

\section{Hypoglycaemia screening versus no screening}

One cohort compared breastfeeding and formula supplementation in the first week in women with and without insulin-dependent diabetes mellitus (IDDM). Infants of women with IDDM had been placed separately in the neonatal unit after birth for observation of hypoglycaemia, though the method of screening was not described.

\begin{tabular}{|c|c|}
\hline Total no of guidelines & $n=12$ \\
\hline \multicolumn{2}{|l|}{ Guideline types $(n=12)$} \\
\hline Sponsored national & $50 \%$ \\
\hline Unsponsored international & $33 \%$ \\
\hline Sponsored international & $17 \%$ \\
\hline \multicolumn{2}{|l|}{ Definition of hypoglycaemia $(n=12)$} \\
\hline Yes & $75 \%$ \\
\hline Unclear & $17 \%$ \\
\hline No & $8 \%$ \\
\hline \multicolumn{2}{|l|}{ Whom to screen } \\
\hline Infant of diabetic mother & $75 \%$ \\
\hline Premature & $75 \%$ \\
\hline Small for gestational age & $66 \%$ \\
\hline Large for gestational age & $58 \%$ \\
\hline Intrapartum asphyxia, sepsis, Rhesus & $8 \%$ each \\
\hline \multicolumn{2}{|l|}{ disease } \\
\hline \multicolumn{2}{|l|}{ Perinatal hypoxia-ischaemia, congenital } \\
\hline \multicolumn{2}{|l|}{$\begin{array}{l}\text { heart disease, infection, hypothermia, intrauterine } \\
\text { growth retardation, maternal risk factors }\end{array}$} \\
\hline Not specified & $8 \%$ \\
\hline \multicolumn{2}{|l|}{ Recommended glucose assays $(n=9)$} \\
\hline $\begin{array}{l}\text { Screen with point-of-care meter; }{ }^{*} \text { confirm with } \\
\text { laboratory } \dagger\end{array}$ & $56 \%$ \\
\hline Point-of-care glucose metre is sufficient ${ }^{\star}$ & $22 \%$ \\
\hline 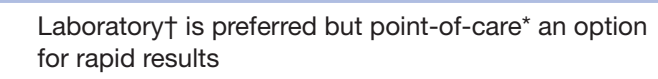 & $11 \%$ \\
\hline Laboratory† should be used for diagnosis & $11 \%$ \\
\hline \multicolumn{2}{|l|}{ Acute harms of hypoglycaemia $(n=5)$} \\
\hline Seizures & $80 \%$ \\
\hline Visual loss, apnoea, cyanosis, coma & $40 \%$ each \\
\hline $\begin{array}{l}\text { Hearing loss, brain injury, lethargy, irritability, } \\
\text { hypotonia, poor suck, tachypnoea, bradycardia, } \\
\text { hypothermia }\end{array}$ & $20 \%$ each \\
\hline \multicolumn{2}{|l|}{ Long-term neurodevelopmental harms $(n=7)$} \\
\hline Cognitive dysfunction & $57 \%$ \\
\hline Motor dysfunction & $43 \%$ \\
\hline Visual dysfunction & $29 \%$ \\
\hline Seizures, unspecified neurodevelopmental harms & $14 \%$ each \\
\hline \multicolumn{2}{|l|}{ Screening harms $(n=7)$} \\
\hline Pain and discomfort & $43 \%$ \\
\hline Breast feeding & $43 \%$ \\
\hline Cost & $14 \%$ \\
\hline
\end{tabular}

*Laboratory methods include: glucose oxidase, hexokinase or dehydrogenase methods.

†For example, glucostix reagent strips, handheld reflectance colorimeter and electrode methods.

\section{Guidelines}

The 12 included guidelines were published between 1997 and 2018 (median 2009: table 3). Seventeen per cent clearly defined a systematic literature search. None specified a funding source. 
Most guidelines (75\%) clearly defined NH. One stated that $\mathrm{NH}$ cannot be described by a single value, and another defined hypoglycaemia as a range of values at which the onset of abnormal symptoms arise (online supplemental table 3). Twenty-five per cent of guidelines $(n=3)$ based the diagnosis of hypoglycaemia on clinical manifestations, while $42 \% \quad(n=5)$ based the severity of hypoglycaemia on clinical manifestations. The most common quantitative thresholds for hypoglycaemic were $<2.2 \mathrm{mmol} / \mathrm{L}$ ( $\mathrm{n}=3$ ) and $<2.6 \mathrm{mmol} / \mathrm{L}(\mathrm{n}=2)$.

The most common reasons for asymptomatic hypoglycaemia screening were maternal diabetes, prematurity, small for GA and large for GA (table 3). Of the $50 \%$ of guidelines that defined large-for-GA and smallfor-GA $(n=6)$, all but one defined small for GA as birth weight below the 10th percentile and large for GA as birth weight above the 90th. Five of the nine guidelines that commented on choice of assay methods suggested screening immediately with a point-of-care glucose metre and confirming results by a laboratory method; the others disagreed (table 3 ).

More guidelines explicitly considered potential harms of hypoglycaemia than potential harms of screening. Five of 12 guidelines (42\%) characterised acute harms of hypoglycaemia including seizures, apnoea, cyanosis, lethargy, irritability, hypotonia, poor sucking, tachypnoea, bradycardia, hypothermia, visual and hearing loss. Seven guidelines $(58 \%)$ described neurodevelopmental harms associated with hypoglycaemia, including seizures and worsening visual, motor and cognitive function. Seven guidelines $(58 \%)$ cited parental concerns about hypoglycaemia screening: most commonly neonatal pain and discomfort, breastfeeding difficulty, and cost. No concerns about parents were mentioned, aside from their need for education.

\section{DISCUSSION}

\section{Screening-related studies}

The primary literature that informs hypoglycaemia screening is a series of studies that relate neurodevelopmental outcomes (especially at preschool ages) to postnatal hypoglycaemia observed at a wide variety of quantitative thresholds. Most studies are of routinely screened babies, though many address more compromised neonates. Although many observational studies described the association between hypoglycaemia and neurodevelopment, only one RCT evaluated different treatment thresholds in terms of their neurodevelopmental outcome. ${ }^{20}$

Other hypoglycaemia-related outcomes included admissions, seizures, and breastfeeding. One study compared (breastfeeding) outcomes of babies who were screened to those who were not screened: but confounded by maternal diabetes, and with a co-intervention of rooming in. $^{21}$

\section{Intervention-related studies}

Aside from the above-mentioned studies, only one other intervention study included neurodevelopment as an outcome, although neurodevelopment justifies intervention: rather, the outcomes of intervention studies generally were intended effects of treatment such as admissions, costs and breast feeding. The RCTs assessed dextrose gels (two), oral sucrose (one) and different glucose-level thresholds of intervention (1); other studied interventions included altered breastfeeding and emphasis on skin to skin.

\section{Guidelines}

Limitations with this evidence complicate the establishment of normative hypoglycaemia thresholds, which varied significantly among guidelines. Guidelines often distinguished symptomatic and asymptomatic hypoglycaemia, but few primary studies did, which limits the epidemiological support for this nuance. Guidelines did not arrive at a consensus definition of postnatal hypoglycaemia. Guidelines mostly agree on screening infants of diabetic mothers and premature babies, though fewer would screen babies at the extremes of birth weight centiles. Guidelines' most commonly cited concerns were long-term neurodevelopmental harm, and acute hypoglycaemia complications. Most guidelines did not discuss potential harms of screening, although implicit in screening only 'at-risk babies' is that although hypoglycaemic occurs in 'normal' babies, ${ }^{22} 23$ screening likely harms more than it helps at some threshold of low risk.

\section{Gaps}

Other gaps emerged from this review. Only one cohort study directly compared outcomes in screened versus unscreened newborns, and did not consider neurodevelopmental outcomes. Many observational studies described the association between hypoglycaemia and neurodevelopment, but only one RCT evaluated different treatment thresholds in terms of their neurodevelopmental outcome at 18 months of age; ideally, such a study would be replicated, and extended to school age to better understand the developmental effects of different thresholds for intervention. ${ }^{11}$

More guidelines addressed potential harms of screening than primary studies, with listed concerns including neonatal pain, breastfeeding difficulty and cost. Further potential complications of screening include lowered maternal confidence in her ability to care for her baby, and maternal anxiety in general. ${ }^{8}$ No study considered the parental perspective on 'routine' glucose monitoring.

Markers of increased pain were associated with decreased brain cortical thickness, and poorer earlychildhood cognitive and internalising behaviour scores in premature babies. ${ }^{24}$ Maternal-infant separation ${ }^{24}$ and maternal anxiety ${ }^{25}$ also appear to have negative effects, possibly causing altered neurodevelopment and cognitive outcomes ${ }^{24}$ and generally less effective parenting behaviours. ${ }^{25}$ Breastfeeding failure increases certain newborn and maternal health risks. ${ }^{26}$ From a risk-benefit perspective, it would be informative to conduct research 
that considers potential harms of screening as well as benefits.

\section{Future research}

It still ${ }^{112728}$ would be informative for future research to better define an optimal threshold for hypoglycaemia that warrants screening and intervention, based on longterm neurodevelopmental outcomes: informed also by a better definition of potential harms of screening. The threshold can be defined in terms of the level of the low glucose, the timing of the low glucose, and the persistence of the low level. ${ }^{20} \mathrm{~A}$ clearer threshold could inform our interpretation of descriptive studies of glucose levels in the context of different risk factors, to inform which risk factors together warrant screening. As literature on oxygenation in prematurity moved towards assessing target oxygen levels, ${ }^{29}$ the $\mathrm{NH}$ literature should describe empirical testing of different glucose targets based on the hypotheses generated from the many observational studies about harms associated with hypoglycaemia: such intervention studies should consider neurodevelopmental outcomes as well as intended ancillary treatment benefits. Also worthy of study would be a comparison of outcomes of screened and unscreened newborns who are potentially at risk, to define the optimal selection of 'at-risk' newborns to screen. Assay and treatment protocols should be described for context: for example, whereas 'gold-standard' glucose assay methods ${ }^{11}$ may optimise hypoglycaemia management, quicker point-ofcare testing could represent routine clinical practice. To optimise the care approach to the whole family, future research should consider the parents' perspective.

\section{Strengths and limitations}

The primary strength of this study was its broad literature search. This review's subsection on neurodevelopmental outcomes and hypoglycaemia included 32 studies and more than 10000 babies, whereas the 2019 systematic review included 11 studies and 4041 infants. ${ }^{11}$ This evidence summary was transparent and systematic in identifying the types of evidence in the field of $\mathrm{NH}^{30}$ However, as a scoping study, this review did not consider study results or assess the quality of the evidence, so it does not provide synthesised answers to inform clinical decision making. ${ }^{30}$ Our analysis also considered only primary study outcomes as opposed to all study outcomes. Finally, language restrictions may have missed a few studies.

\section{CONCLUSION}

The lack of consensus among guidelines on NH screening and treatment reflects the nature of the background literature. The primary literature that informs hypoglycaemia screening is a series of studies that relate neurodevelopmental outcomes to postnatal hypoglycaemia. However, $\mathrm{NH}$-intervention-study outcomes generally were intended effects of treatment such as cost and breastfeeding: there is much less literature on NH treatment from the neurodevelopmental perspective, which justifies $\mathrm{NH}$ treatment. Also, guidelines addressed potential harms of screening more often than primary studies. Thus, given the type of evidence that exists on the management of $\mathrm{NH}$, clinicians can anticipate ongoing controversy and uncertainty around $\mathrm{NH}$ screening and treatment. The above-proposed research could inform an evidence-based targeting of hypoglycaemia screening and treatment to babies in whom the risk justifies intervention, for this common newborn problem.

Contributors LM, AM and JH helped design the data collection instrument, ran the software, extracted data, carried out the first content analyses, and wrote the first draft of the results section. AM also obtained funding for his studentship. Overall, LM, AM and JH made equal contributions to the project; as authors, they were listed alphabetically. VAW conceptualised and designed the study methodology, contributed to project supervision, and helped acquire project funding. AH designed and carried out the electronic literature search, drafted the literature search methodology, and contributed to the analysis. MN provided input regarding the design of study methods (in particular with regard to the electronic literature search and data extraction). AG contributed to the development of study methods with regard to data extraction; administratively in training the data extractors regarding content analysis; and helped acquire funding. BS contributed methodologically with input into data extraction and the design of the electronic literature search. MS conceptualised and designed the study; coordinated and supervised data collection; analysed the data; drafted the introduction, methods and discussion sections; and acquired funding for the project. All authors revised the manuscript critically for important intellectual content. All authors approved the final manuscript as submitted and agree to be accountable for all aspects of the work. MS had access to the data, controlled the decision to publish, and accepts full responsibility for the conduct of the study as guarantor.

Funding The study was supported by a grant from the Innovation Fund Provincial Oversight Committee. AM was supported by a University of Ottawa Faculty of Medicine Summer Studentship Award.

Competing interests None declared.

Patient consent for publication Not applicable.

Ethics approval As a secondary analysis of published data, this study did not require ethics approval.

Provenance and peer review Not commissioned; externally peer reviewed.

Data availability statement Data are available in a public, open access repository. Dataset available from the 0pen Science Framework repository at https://osf.io/ vu3c4/. DOI 10.17605/OSF.IO/VU3C4.

Supplemental material This content has been supplied by the author(s). It has not been vetted by BMJ Publishing Group Limited (BMJ) and may not have been peer-reviewed. Any opinions or recommendations discussed are solely those of the author(s) and are not endorsed by BMJ. BMJ disclaims all liability and responsibility arising from any reliance placed on the content. Where the content includes any translated material, BMJ does not warrant the accuracy and reliability of the translations (including but not limited to local regulations, clinical guidelines, terminology, drug names and drug dosages), and is not responsible for any error and/or omissions arising from translation and adaptation or otherwise.

Open access This is an open access article distributed in accordance with the Creative Commons Attribution Non Commercial (CC BY-NC 4.0) license, which permits others to distribute, remix, adapt, build upon this work noncommercially, and license their derivative works on different terms, provided the original work is properly cited, appropriate credit is given, any changes made indicated, and the use is non-commercial. See: http://creativecommons.org/ licenses/by-nc/4.0/.

\section{ORCID iDs}

Linda Mardiros http://orcid.org/0000-0002-5126-0641 Ahmed Musa http://orcid.org/0000-0003-0590-6170

Vivian A Welch http://orcid.org/0000-0002-5238-7097

Amanda Hodgson http://orcid.org/0000-0002-9750-6651 


\section{REFERENCES}

1 Government of Canada. Maternal diabetes in Canada. table 1: temporal trends in rates (per 1,000 deliveries) of maternal diabetes in Canada, 2004/05-2010/11. Available: https://www.canada.ca/en/ public-health/services/publications/healthy-living/maternal-diabetescanada.html [Accessed 22 Oct 2019].

2 Loftin RW, Habli M, Snyder CC, et al. Late preterm birth. Rev Obstet Gynecol 2010;3:10-19.

3 Stewart CE, Sage ELM, Reynolds P. Supporting 'Baby Friendly': a quality improvement initiative for the management of transitional neonatal hypoglycaemia. Arch Dis Child Fetal Neonatal Ed 2016;101:F344-7.

4 Sundercombe SL, Raynes-Greenow CH, Carberry AE, et al. Audit of a clinical guideline for neonatal hypoglycaemia screening. J Paediatr Child Health 2013;49:833-8.

5 Harris DL, Weston PJ, Harding JE. Incidence of neonatal hypoglycemia in babies identified as at risk. $J$ Pediatr 2012;161:787-91.

6 Simmons R, Stanley C. Neonatal hypoglycemia studies - is there a sweet story of success yet? N Engl J Med Overseas Ed 2015;373:1567-9.

7 Narvey MR, Marks SD. The screening and management of newborns at risk for low blood glucose. Paediatr Child Health 2019;24:536-44.

8 Haninger NC, Farley CL. Screening for hypoglycemia in healthy term neonates: effects on breastfeeding. J Midwifery Womens Health 2001;46:292-8

9 Acharya K, Ackerman PD, Ross LF. Pediatricians' attitudes toward expanding newborn screening. Pediatrics 2005;116:e476-84.

10 Shah R, Harding J, Brown J, et al. Neonatal glycaemia and neurodevelopmental outcomes: a systematic review and metaanalysis. Neonatology 2019;115:116-26.

11 Hay WW, Raju TN, Higgins RD, et al. Knowledge gaps and research needs for understanding and treating neonatal hypoglycemia: workshop report from Eunice Kennedy Shriver National Institute of child health and human development. J Pediatr 2009;155:612-7.

12 Arksey H, O'Malley L. Scoping studies: towards a methodological framework. Int J Soc Res Methodol 2005;8:19-32.

13 Tricco AC, Lillie E, Zarin W, et al. PRISMA extension for scoping reviews (PRISMA-ScR): checklist and explanation. Ann Intern Med 2018;169:467-73.

14 Mardiros L, Saginur M, Musa A. Data from: Newborn Hypoglycemia Screening - A Scoping Review. Open Science Framework 2020.

15 McGowan J, Sampson M, Salzwedel DM, et al. PRESS Peer Review of Electronic Search Strategies: 2015 Guideline Statement. J Clin Epidemiol 2016;75:40-6.
16 Canadian Agency for Drugs and Technologies in Health. Strings Attached: CADTH's Database Search Filters. Available: https://cadth. $\mathrm{ca} /$ resources/finding-evidence/strings-attached-cadths-databasesearch-filters

17 Wong JJ, Côté P, Sutton DA, et al. Clinical practice guidelines for the noninvasive management of low back pain: a systematic review by the Ontario protocol for traffic injury management (optima) collaboration. European Journal of Pain 2017;21:201-16.

18 Covidence systematic review software, veritas health innovation, Melbourne, Australia. Available: www.covidence.org

19 Brouwers MC, Kho ME, Browman GP, et al. Agree II: advancing Guideline development, reporting and evaluation in health care. Can Med Assoc J 2010;182:E839-42.

20 van Kempen AAMW, Eskes PF, Nuytemans DHGM, et al. Lower versus traditional treatment threshold for neonatal hypoglycemia. $N$ Engl J Med 2020;382:534-44.

21 Ferris AM, Neubauer SH, Bendel RB, et al. Perinatal lactation protocol and outcome in mothers with and without insulin-dependent diabetes mellitus. Am J Clin Nutr 1993;58:43-8.

22 Hoseth E, Joergensen A, Ebbesen F. Blood glucose levels in a population of healthy, breast fed, term infants of appropriate size for gestational age. Arch Dis Child Fetal Neonatal Ed 2000;83:117F-9.

23 Nicholl R. What is the normal range of blood glucose concentrations in healthy term newborns? Arch Dis Child 2003;88:238-9.

24 Mooney-Leber SM, Brummelte S. Neonatal pain and reduced maternal care: early-life stressors interacting to impact brain and behavioral development. Neuroscience 2017;342:21-36.

25 Seymour M, Giallo R, Cooklin A, et al. Maternal anxiety, risk factors and parenting in the first post-natal year. Child Care Health Dev 2015;41:314-23.

26 Victora CG, Bahl R, Barros AJD, et al. Breastfeeding in the 21st century: epidemiology, mechanisms, and lifelong effect. The Lancet 2016;387:475-90.

27 Boluyt N, van Kempen A, Offringa M. Neurodevelopment after neonatal hypoglycemia: a systematic review and design of an optimal future study. Pediatrics 2006;117:2231-43.

28 Vain NE, Chiarelli F. Neonatal hypoglycaemia: a never-ending story? Neonatology 2021;118:522-9.

29 Askie LM, Darlow BA, Finer N, et al. Association between oxygen saturation targeting and death or disability in extremely preterm infants in the neonatal oxygenation prospective meta-analysis collaboration. JAMA 2018;319:2190-201.

30 Munn Z, Peters MDJ, Stern C, et al. Systematic review or scoping review? guidance for authors when choosing between a systematic or scoping review approach. BMC Med Res Methodol 2018;18:143. 